\title{
A Non-Invasive Bone Fracture Monitoring Analysis using an UHF Antenna
}

\author{
Mahreen Riaz \\ Biomedical Engineering and \\ Communications (BiMEC) research \\ Centre \\ London South Bank University \\ London U.K. \\ riazm4@1sbu.ac.uk \\ Mohammad Ghavami \\ Biomedical Engineering and \\ Communications (BiMEC) research \\ Centre \\ London South Bank University \\ London U.K. \\ ghavamim@1sbu.ac.uk
}

\author{
Gianluigi Tiberi \\ Biomedical Engineering and \\ Communications (BiMEC) research \\ Centre \\ London South Bank University \\ London U.K. \\ tiberig@1sbu.ac.uk \\ Sandra Dudley \\ Biomedical Engineering and \\ Communications (BiMEC) research \\ Centre \\ London South Bank University \\ London U.K. \\ dudleyms@1sbu.ac.uk
}

\author{
Humphrey Asani \\ Biomedical Engineering and \\ Communications (BiMEC) research \\ Centre \\ London South Bank University \\ London U.K. \\ batierah@1sbu.ac.uk
}

\begin{abstract}
This paper shows the design of an UHF antenna which can be used for monitoring a bone fracture. A simple cylindrical model is proposed to replicate the properties of bone with an inclusion representing a blood-mimicking lesions, indicating bone fracture. The proposed dipole antenna with dimensions of $150 \mathrm{~mm} \times 110 \mathrm{~mm}$ is analyzed into CST Design Studio to show the s-parameters variations to indicate the presence of fracture. Two identical antennas are used on the top and bottom of the bone. The proposed method may serve the purpose of a low cost and non-invasive procedure for fracture monitoring.
\end{abstract}

Keywords-UHF, antenna, bone fracture, dipole, phantom, non-invasive.

\section{INTRODUCTION}

Bone fractures are categorised as the most common traumas in musculoskeletal injury. To analyse bone fracture, there are various techniques practiced across all the hospitals worldwide. The most commonly used method to analyse is $\mathrm{x}-$ ray in which the location and severity of the fracture is obtained by visually examining the image. Radiologists often experience problem in analysis due to imaging quality and various anatomical structures overlapping; To solve this problem, advanced computer vision and several techniques of machine learning are introduced to improve the existing method [1]. However, using x-ray for bone fractures monitoring is not feasible.

In past decades, implanted medical devices have grown interest in the research field of health care monitoring. Medical applications shown in the literature mostly operate in the radio frequency (RF) band or in the medical device and radio communication service (MedRadio) at 401-406 $\mathrm{MHz}$ [2 -4 ] or at $2.44-2.46 \mathrm{MHz}$ [5 - 7] in the ISM band. Mostly the implanted antennas are placed under the skin or in the fat and muscle tissues. Due to body's highly complex structure and lossy properties of tissues, it is very challenging to design implanting devices; in addition, biocompatibility may be an issue [8].

Non-invasive sensors are most advantageous for monitoring purposes (such as patients' behaviour, routine activities and physiological functionalities), as they provide comfort, robustness and a high level of safety from infection and cross contamination $[9,10]$. Despite all the advancements in medical industry, wearable devices implementation, specifically on body radiators is not an easy challenge. There are chances of performance degradation due to dielectric coupling, and power absorption by human body $[11,12]$ and interferences effects.

There could be few constraints in UHF RFID setup due to interference with mobile communication channel. The UHF RFID specification and standards are defined by EPC global Class 1, Gen2 [13] which defines various standards such as ISO 29143 air interface for RFID mobile integrators, ISO 18000-6C and ISO 18000-6A for direct mode operation [14]. Mobile phones could be a possible source of interference due to two close frequency band to UHF RFID system. The 800 $\mathrm{MHz}$ band is used in Spain and other EU countries for 4G LTE use and $900 \mathrm{MHz}$ band for 3G GSM in rural areas. The proximity of UHF RFID band usage for short range devices may cause some interference effects, causing in degradation of radio signals behaviour. This could be more obvious in the upper adjacent band due to out-of-band (OOB) emissions generated by wide band LTE systems [15].

This work is introducing a novel approach of monitoring the bone fracture non-invasively by using 50-ohm UHF antennas. Two identical antennas are placed on the top and bottom side of the forearm and the s-parameters variation based on the location of fracture helps in the identification process. A simple cylindrical model to mimic an arm structure is considered for preliminary investigation. 


\section{ANTENNA DESIGN}

A UHF antenna having dimensions of $41 \mathrm{~mm} \times 127.81$ $\mathrm{mm}$ is proposed as shown in Figure 1. The antenna structure is dual dipole and designed on PET substrate having thickness

of $0.05 \mathrm{~mm}$ and the metal tracks are made up of aluminium material with $0.01 \mathrm{~mm}$ thickness. UHF frequency band is usually included in the band employed when performing microwave bone imaging, since it guarantees good penetration and resolution [16].

The dimensions of the antenna structure are chosen to resonate at UHF frequency band region $[860-960 \mathrm{MHz}]$ as shown in Figure 2. It can be seen that a low power reflection co-efficient of $-16.84 \mathrm{~dB}$ is achieved at $866 \mathrm{MHz}$ indicating chances of more radiation at resonance frequency [17]. The reason of choosing UHF frequency band is satisfactory achievement of resonance and observation of distinct characteristics for power reflection and power transmission coefficients to observe the variation in inclusion monitoring. The gain of the antenna measured at $866 \mathrm{MHz}$ is $1.72 \mathrm{~dB}$ relative to an isotropic antenna indicates a good signal strength of the antenna which can be sent or receive in a specific direction. as shown in Figure 3. The radiation pattern also shows a high far field radiation range. Two identical antennas are simulated into the software for the proposed fracture monitoring process.

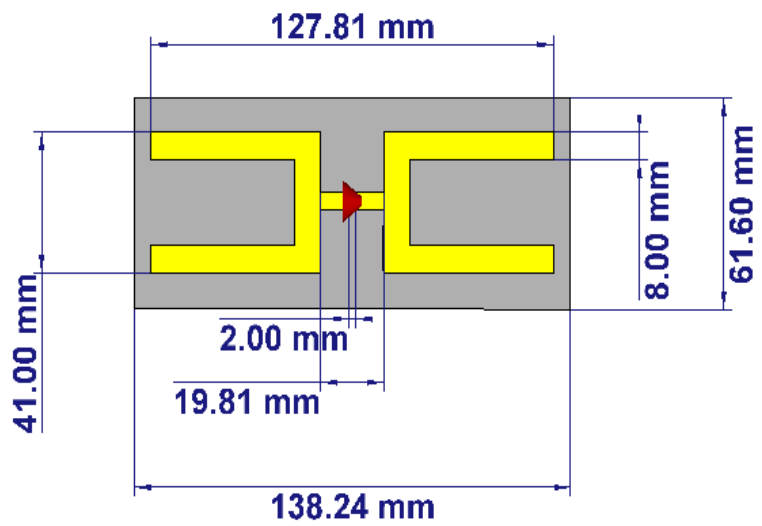

Figure 1: UHF antenna geometry.

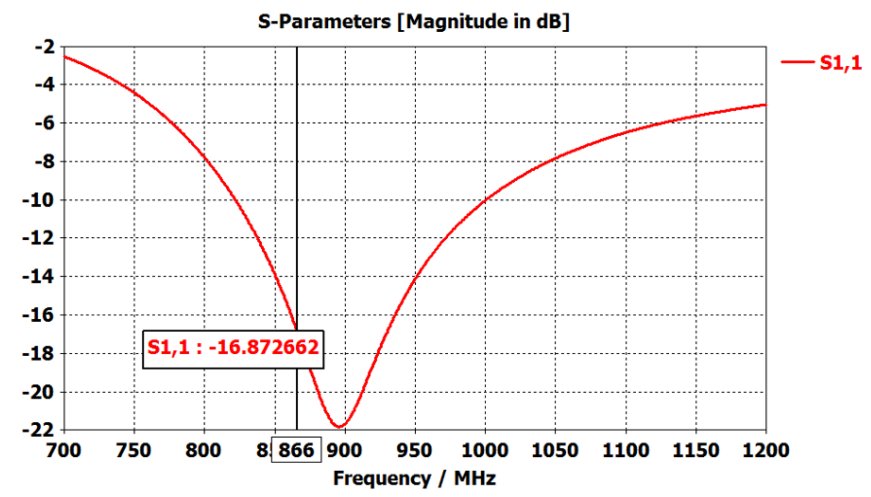

Figure 2: Power reflection coefficient graph.

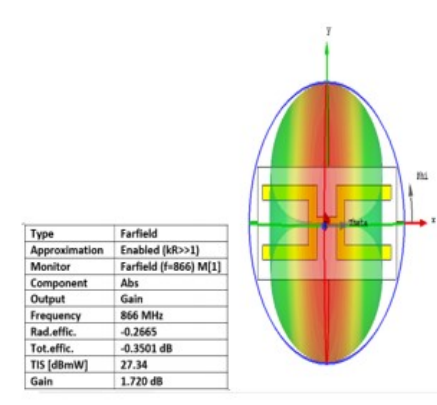

Figure 3: 3D gain of the proposed antenna.

\section{BONE FractURE PHANTOM PROPERTIES}

A phantom is designed into CST Microwave studio to mimic the bone fracture as shown in Figure 4. A cylindrical model is used to mimic the arm structure. A simple model is proposed with the dimensions of the bone as $110 \mathrm{~mm} \times 150$ $\mathrm{mm}$ and the properties at UHF shown in Table 1. To replicate the fracture, a cylindrical model having dimensions of $15 \mathrm{~mm}$ $\mathrm{x} 70 \mathrm{~mm}$ is used to indicate blood mimicking lesion with the properties at UHF shown in the Table 1 [18].

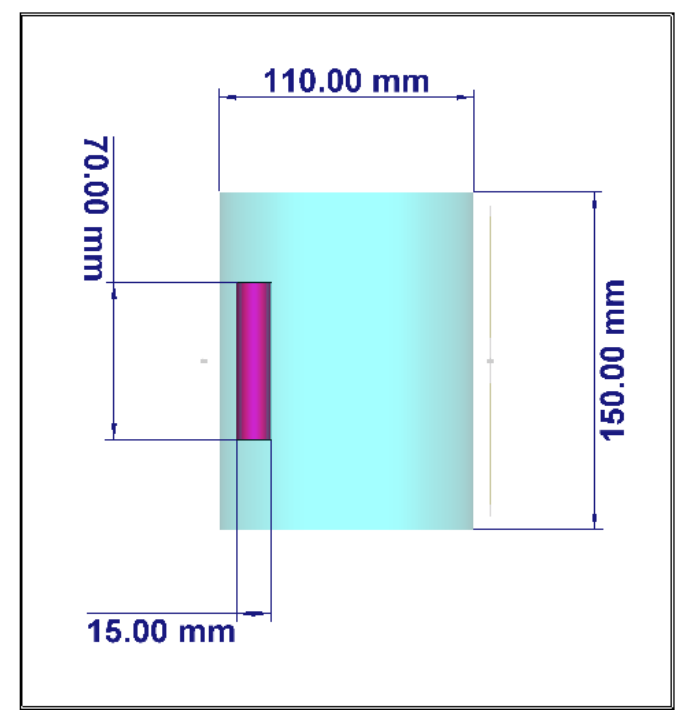

Figure 4: Cylindrical model of bone fracture.

TABLE I. Properties OF BONE FRACTURE PHANTOM

\begin{tabular}{|l|l|c|}
\hline \multirow{2}{*}{\multicolumn{1}{|c|}{ Properties }} & \multicolumn{2}{|c|}{ Model of bone fracture phantom } \\
\cline { 2 - 3 } & \multicolumn{1}{|c|}{ Bone } & Fracture (blood) \\
\hline $\begin{array}{l}\text { Dielectric } \\
\text { Constant }[\varepsilon r]\end{array}$ & 6.25 & 80 \\
\hline Mue $[\mu]$ & 1 & 1 \\
\hline $\begin{array}{l}\text { Electrical } \\
\text { conductivity } \\
{[\mathrm{S} / \mathrm{m}]}\end{array}$ & 0.28 & 2.19 \\
\hline
\end{tabular}




\section{SimULATION RESULTS OF NORAMAL AND FRACTURED BONE}

In Figure 5, a simulation model is shown with the normal bone having a radius of $55 \mathrm{~mm}$ and a height of $150 \mathrm{~mm}$. Two UHF proposed antennas are used to detect the fracture by placing on the top and on the bottom side of arm structure. For fracture monitoring, a blood-mimicking cylindrical inclusion is introduced having a radius of radius of $7.5 \mathrm{~mm}$ and a height of of $70 \mathrm{~mm}$. The inclusion is positioned at various locations of the bone structure to analyze the effect of variable locations on the s-parameters and to observe the changes.

For this reason, three locations are chosen starting with the inclusion on the left side as shown in the Figure 6 and categorized as location 1. The second location is the center of the bone where the blood inclusion is introduced and shown in Figure 7. The last location is the right location no. 3 with the inclusion and shown in the Figure 8.

Now, s- parameters are analysed to observe the changes without and with bone fracture monitoring at various locations. Figure 9 shows the power reflection coefficient $\mathrm{S} 1,1$ (that indicates how much power is reflection back from antenna 1) and the S2,2 (that shows the amount of power reflected from antenna 2). Figure 10 shows the power transmission S1,2 (from antenna 2 to antenna 1), which is the same as S2,1. from Fig 9 and Fig 10 it is observed:

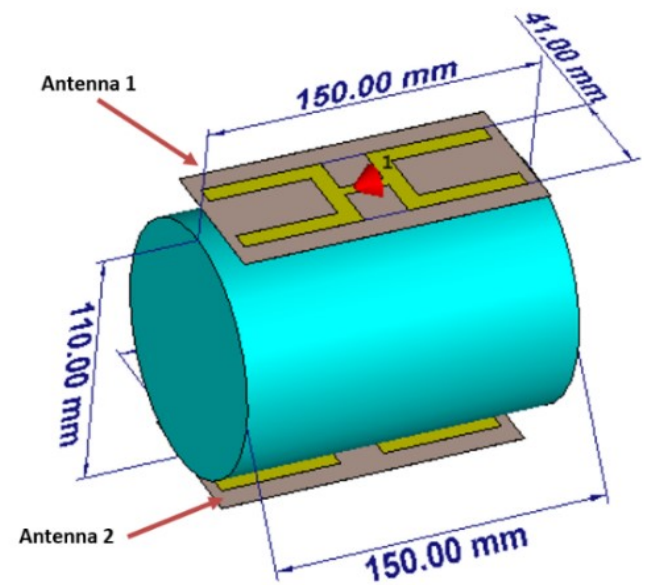

Figure 5: Normal bone with two UHF detection antennas.

I. In case of no inclusion, $\mathrm{S} 1,1$ and $\mathrm{S} 2,2$ of both antennas are similar. Specifically, the values show the power reflection coefficient of $-26 \mathrm{~dB}$ and -33.5 $\mathrm{dB}$ for antenna 1 and antenna 2 respectively. The slight difference between the S1,1 and S2,2 may be related to the mesh grid asymmetries.

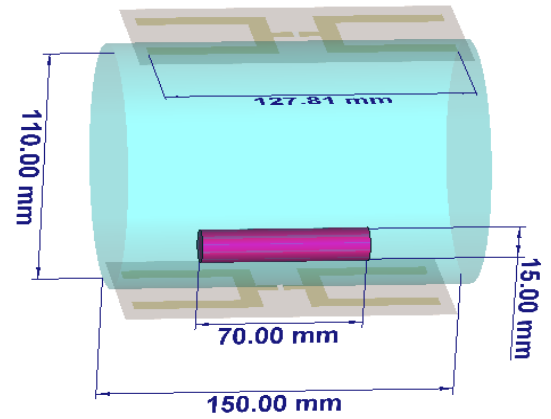

Figure 6: Bone fracture inclusion on the left sidelocation 1.

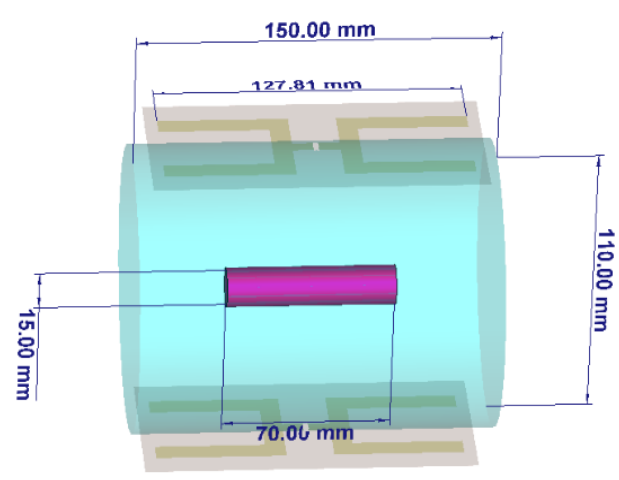

Figure 7: Bone fracture inclusion in the centre- location 2.

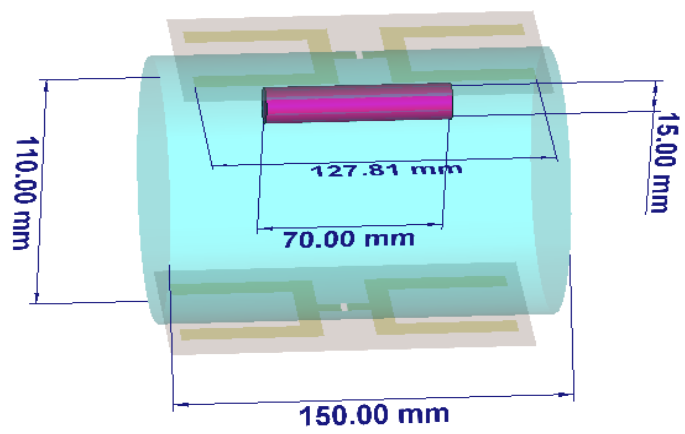

Figure 8: Bone fracture inclusion on right side- location 3.

II. S1,1 and S2,2 when the inclusion is positioned on the left are $-31 \mathrm{~dB}$ and $-15 \mathrm{~dB}$, respectively. This high difference may be explained noting that the inclusion is near to antenna 2, and thus the inclusion enhances the power reflected to antenna 2 . 


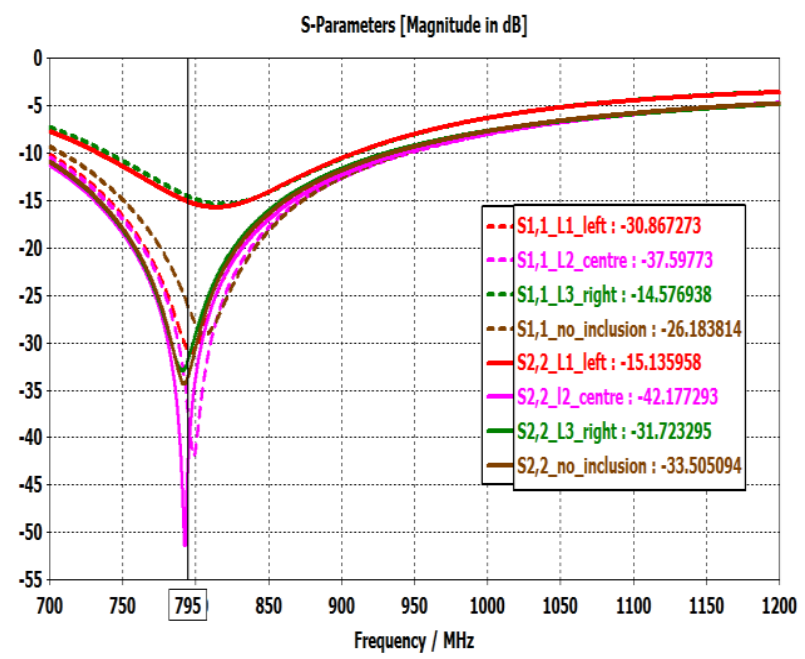

Figure 9: Power reflection coefficient- S1,1.

III. S1,1 and S2,2 when the inclusion is positioned on the centre are $-37.5 \mathrm{~dB}$ and $-42 \mathrm{~dB}$, respectively. Such values are quite similar, and this is due to the fact that the antennas are equidistant from the central location of inclusion.

IV. In addition, it can be seen that $\mathrm{S} 1,1$ and $\mathrm{S} 2,2$ show variations from no inclusion to inclusion positioned on the centre, indicating the presence of bone lesion and appreciating the difference from non-fracture to fractured bone.

V. S1,1 and S2,2 when the inclusion is positioned on the right are $-14.5 \mathrm{~dB}$ and $-33.5 \mathrm{~dB}$, respectively. This difference may be explained noting that the inclusion is now closed to antenna 1 , and thus the inclusion enhances the power reflected to antenna 1.

VI. Concerning S1,2 the presence of the inclusion reduces the transmitted power of approximately 1 $\mathrm{dB}$ when the inclusion is in the centre, and of approximately $2 \mathrm{~dB}$ when the inclusion is near to one of the two antennas.

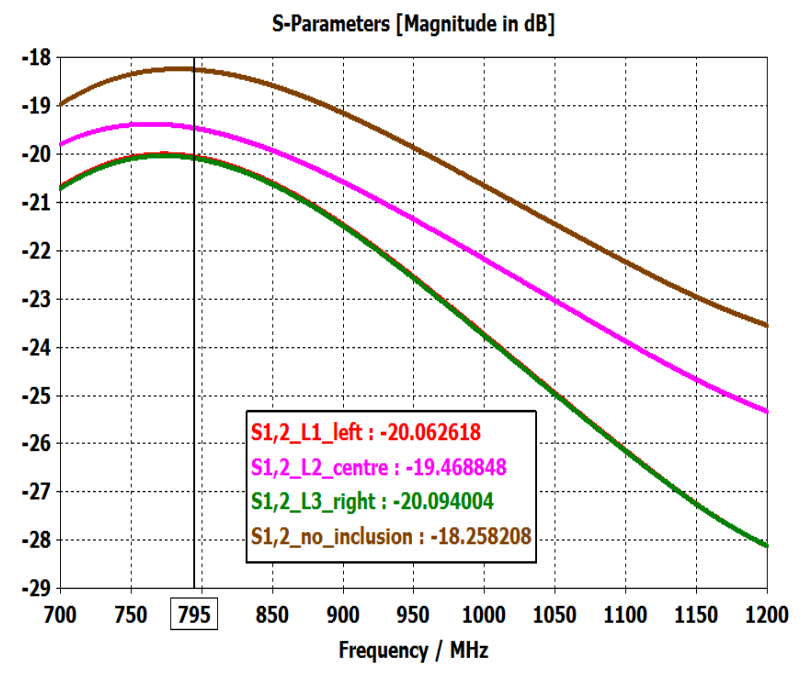

Figure 10: Power transmission coefficient- S1,2.

\section{CONCLUSION}

It can be seen from the comparison result analysis that two identical UHF antennas may serve the purpose of monitoring a bone lesion by considering S-parameters. Reflection and transmission coefficients could be categorized into different levels at UHF frequency band, and such levels may be used for performing a non-invasive bone fracture monitoring.

The proposed method can be useful for non-invasive bone fracture analysis and monitoring. In this context, further steps will involve the investigation of polarization, the simulation of more realistic model (considering different body layers such as skin, muscles, bone, fat, blood and different kind of inclusions), the fabrication of dedicated phantoms to be tested in anechoic camber/realistic scenario, S-parameters characterizations for lesions recovery stages. The impact of interference of mobile communication band with UHF systems should be considered and addressed.

\section{ACKNOWLEDGEMENT}

This project leading to this application has received funding from the European Union's Horizon 2020 research and innovation programme under the Marie Sklodowska-Curie grant agreement No. 793449. This project has received funding from the European Union's Horizon 2020 research and innovation programme under the Marie SklodowskaCurie grant agreement No. 872752.

\section{REFERENCES}

[1] Office of the Surgeon General US. "Bone health and osteoporosis: a report of the Surgeon General." (2004).

[2] Kim, Jaehoon, and Yahya Rahmat-Samii. "Implanted antennas inside a human body: Simulations, designs, and characterizations." IEEE Transactions on microwave theory and techniques 52.8 (2004): 19341943.

[3] Soontornpipit, Pichitpong, Cynthia M. Furse, and You Chung Chung. "Design of implantable microstrip antenna for communication with medical implants." IEEE Transactions on Microwave theory and techniques 52.8 (2004): 1944-1951.

[4] Karacolak, Tutku, Aaron Z. Hood, and Erdem Topsakal. "Design of a dual-band implantable antenna and development of skin mimicking gels for continuous glucose monitoring." IEEE Transactions on Microwave Theory and Techniques 56.4 (2008): 1001-1008.

[5] Xia, Wei, et al. "Performances of an implanted cavity slot antenna embedded in the human arm." IEEE Transactions on Antennas and Propagation 57.4 (2009): 894-899.

[6] Warty, Ruchi, et al. "Characterization of implantable antennas for intracranial pressure monitoring: Reflection by and transmission through a scalp phantom." IEEE Transactions on Microwave Theory and Techniques 56.10 (2008): 2366-2376.

[7] Soora, Shruthi, et al. "A comparison of two and three dimensional dipole antennas for an implantable retinal prosthesis." IEEE Transactions on Antennas and Propagation56.3 (2008): 622-629.

[8] Abadia, Javier, et al. "3D-spiral small antenna design and realization for biomedical telemetry in the MICS band." Radioengineering 18.4 (2009): 359-367.

[9] Soh, Ping Jack, et al. "Wearable wireless health monitoring: Current developments, challenges, and future trends." IEEE Microwave Magazine 16.4 (2015): 55-70.

[10] Foerster, Friedrich, Manfred Smeja, and Jochen Fahrenberg. "Detection of posture and motion by accelerometry: a validation study in ambulatory monitoring." Computers in human behavior 15.5 (1999): 571-583. 
[11] Soh, Ping Jack, et al. "Characterization of a simple broadband textile planar inverted-F antenna (PIFA) for on body communications." 2010 Conference Proceedings ICECom, 20th International Conference on Applied Electromagnetics and Communications. IEEE, 2010.

[12] Soh, Ping Jack, G. A. E. Vandenbosch, and DMM-P. Schreurs. "Onbody characterization of textile antennas for biomedical health monitoring systems." 2013 IEEE topical conference on biomedical wireless technologies, networks, and sensing systems. IEEE, 2013.

[13] Global, E. P. C. "EPC radio-frequency identity protocols class-1 generation-2 UHF RFID protocol for communications at $860 \mathrm{MHz}-$ 960 MHz." Version 1.0 (2008): 23.

[14] ISO/IEC 18000-63:2015, Information Technology—Radio Frequency Identification for Item Management-Part 63:Parameters for Air Interface Communications at $860 \mathrm{MHz}$ to $960 \mathrm{MHz}$ Type C, ANSI, Lawrenceville, NJ, USA, Oct. 2015.

[15] Cairó, Josep-Ignasi, et al. "Interference sources in congested environments and its effects in UHF-RFID systems: A review." IEEE Journal of Radio Frequency Identification 2.1 (2018): 1-8.
[16] Ruvio, G., Cuccaro, A., Solimene, R., Brancaccio, A., Basile, B., \& Ammann, M. J. (2016). "Microwave bone imaging: a preliminary scanning system for proof-of-concept". Healthcare technology letters, 3(3), 218-221.

[17] Riaz, Mahreen, et al. "A novel design of UHF RFID passive tag antenna targeting smart cards limited area." 2018 IEEE International Conference on Consumer Electronics (ICCE). IEEE, 2018.

[18] Gabriel, C., Gabriel, S., \& Corthout, Y. E. (1996). The dielectric properties of biological tissues: I. Literature survey. Physics in medicine \& biology, 41(11), 2231. 